\title{
Many eyes on Earth
}

\section{Swarms of small satellites set to deliver close to real-time imagery of swathes of the planet.}

\section{BY DECLAN BUTLER}

I magine using Google Earth or other online mapping tools to zoom in on high-resolution satellite images of the planet taken just hours or days ago. Navigating backwards and forwards in time, one could track changes in everything from crops, forests and wildlife movement to urban sprawl and natural disasters, all with unrivalled temporal precision.

This is the vision of two Californian startup companies that are set to launch swarms of small imaging satellites, which, by virtue of their sheer numbers, will be able to revisit and photograph huge swathes of the planet as often as several times each day - a frequency much higher than that achieved by current Earthobserving satellites.

San Francisco-based Planet Labs, founded in 2010 by three former NASA scientists, is scheduled to launch 28 of its 'Doves' on 8 January. Each toaster-sized device weighs about 5 kilograms and can take images at a resolution of 3-5 metres.

At Skybox Imaging in nearby Palo Alto, plans are afoot for a swarm of 24 satellites, each weighing about 100 kilograms, which will take images of 1 metre resolution or better. Skybox launched its first satellite on 21 November and plans to launch another this year, followed by the remainder between 2015 and 2017.

In a first - at least for civilian satellites - Skybox's devices will also stream short segments of near-live high-resolution video footage of the planet. So, too, will UrtheCast,

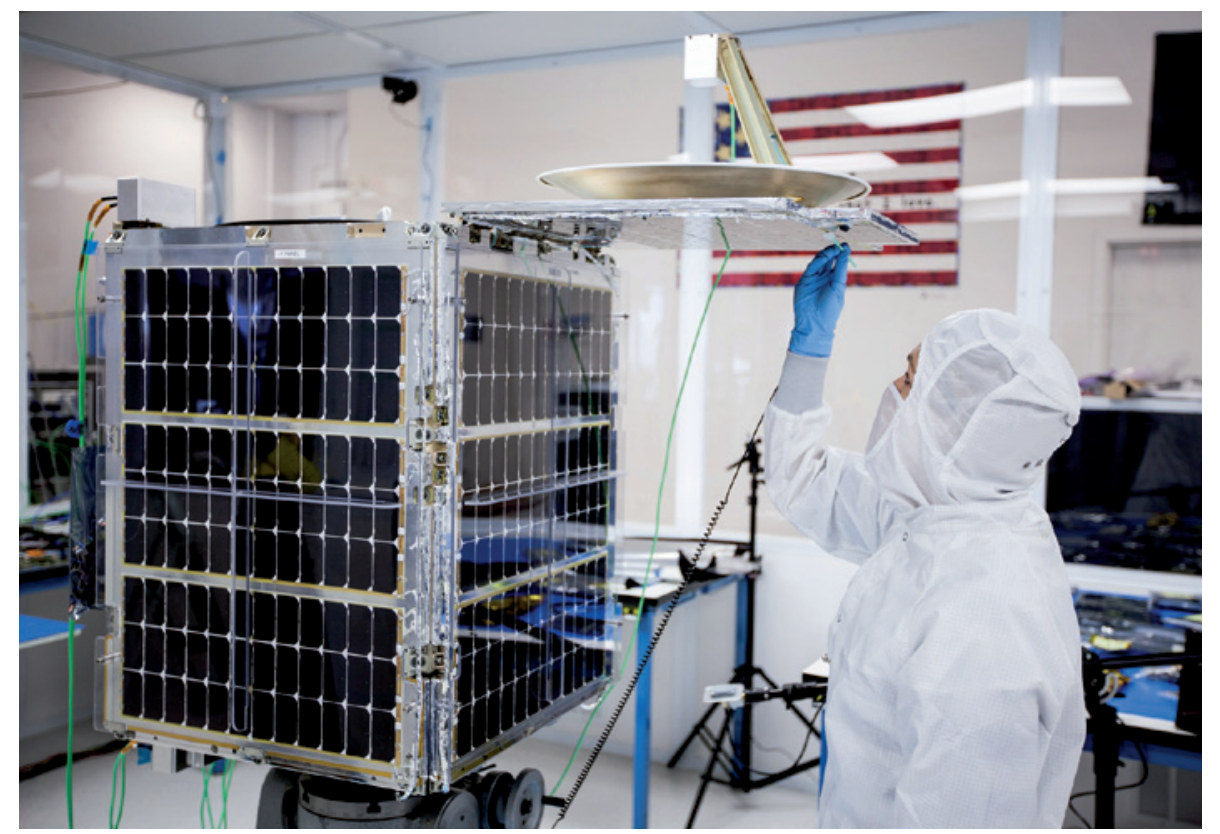

Skybox Imaging plans to launch 24 high-resolution SkySats in the next few years. a start-up based in Vancouver, Canada, whose cameras will hitch a ride on the International Space Station (see go.nature.com/cebdkb).

The efforts could herald a sea change for imaging. Conventional imaging satellites, which are the size of a van and weigh tonnes, cost hundreds of millions of dollars to build and launch (see 'The swarm cometh'). As a result, there are only a handful of operators, and the commercial world fleet comprises

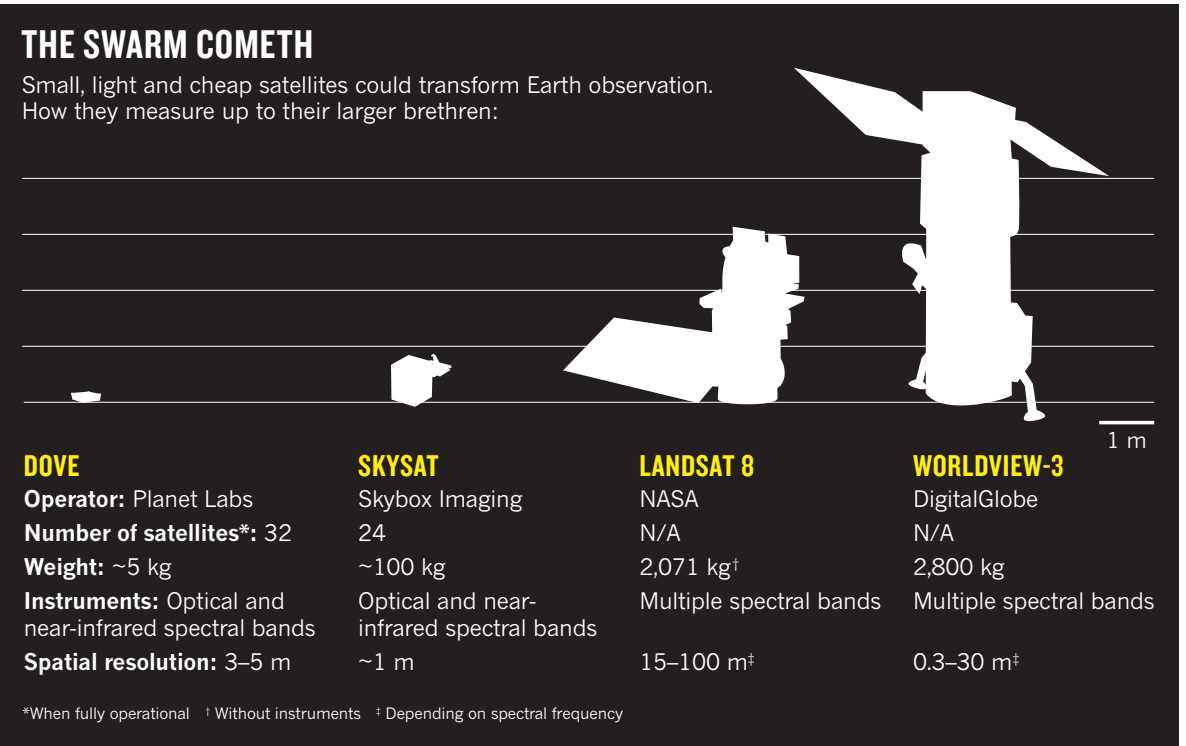

fewer than 20 satellites. Commercial satellites also tend to take pictures mainly when their operators receive orders from customers.

By contrast, the swarm satellites' cameras will always be on, photographing everything in their path and, owing to their numbers, will pass over the same points on Earth with a frequency of hours to a few days, depending on latitude.

The biggest customers of conventional commercial imaging satellites are governments, in particular intelligence agencies and the military. Prices can be prohibitive for many other potential users, including researchers, in areas as diverse as farming, forest carbon management, regional and local planning, and environmental stewardship. By making their images cheaper, the new entrants into the marketplace hope to spur a proliferation of innovative uses. They also hope to offer heavy discounts or even make imagery free to academics and non-governmental organizations.

"This sector has for so long been driven by government requirements and, to a lesser extent, big industry players, that the massmarket consumer - the long tail — has been almost completely neglected," says Scott Larson, chief executive of UrtheCast. Cheaper imagery, he says, will lead to "the democratization of near-real-time Earth-observation data".

To slash costs, Planet Labs and Skybox Imaging use off-the-shelf technologies from the 
- automotive, smartphone and other consumer industries - including low-cost electronics, and sensors from high-end digital cameras. Using the latest technologies from these fast-paced industries also allows the rapid, continuous development of better and better satellites, says Will Marshall, chief executive of Planet Labs. And miniaturizing satellites reduces launch costs.

Because the swarms are still to be launched, scientists have yet to fully assess the quality of the imagery. But the satellites' spatial resolutions of 1-5 metres are much higher than those of most scientific satellites. Landsat, NASA's Earth-observation workhorse, for example, has a resolution of 15-100 metres depending on the spectral frequency, with 30 metres in the visiblelight range.

Such medium-resolution imagery is adequate for many purposes, but higher resolution can have benefits, says Dan Berkenstock, co-founder and chief product officer of Skybox Imaging. He points to a study published in November that found that the use of moderate-resolution Landsat imagery greatly underestimated forest loss in the Democratic Republic of the Congo (A. Tyukavina et al. Environ. Res. Lett. 8, 044039; 2013).

Precision agriculture, a method that uses remote sensing to aid farm management, will also benefit from swarms, says Berkenstock, because the technology will be able to provide timely crop-yield and health estimates down to the level of rows of plants. Such detail could inform decisions on fertilizer and irrigation use, but is currently out of reach of most farmers.

However, spatial resolution is only part of the picture, says Mike Wulder, a researcher at the Canadian Forest Service in Victoria and a member of the Landsat science team. He uses remote sensing to study forests, and notes that good spectral and radiometric resolution (detection of small differences in wavelength and radiation, respectively), are essential for quantitative scientific analyses. "These very small satellites should not be expected to provide data that are similar or in competition with full-blown Earthobserving satellites," he says. "They occupy a different niche."

The scientific value of the swarm data will be "radically dependent" on quality issues, says Greg Asner, an Earth scientist at the Carnegie Institution for Science in Stanford, California. Stitching together such frequent-repeat imagery from so many satellites will be challenging, because performance will probably differ between satellites and vary over time, he argues.

But he is nonetheless excited at the prospect of constantly updated fresh imagery. "It will almost be like updating Google Earth each day," he says. -

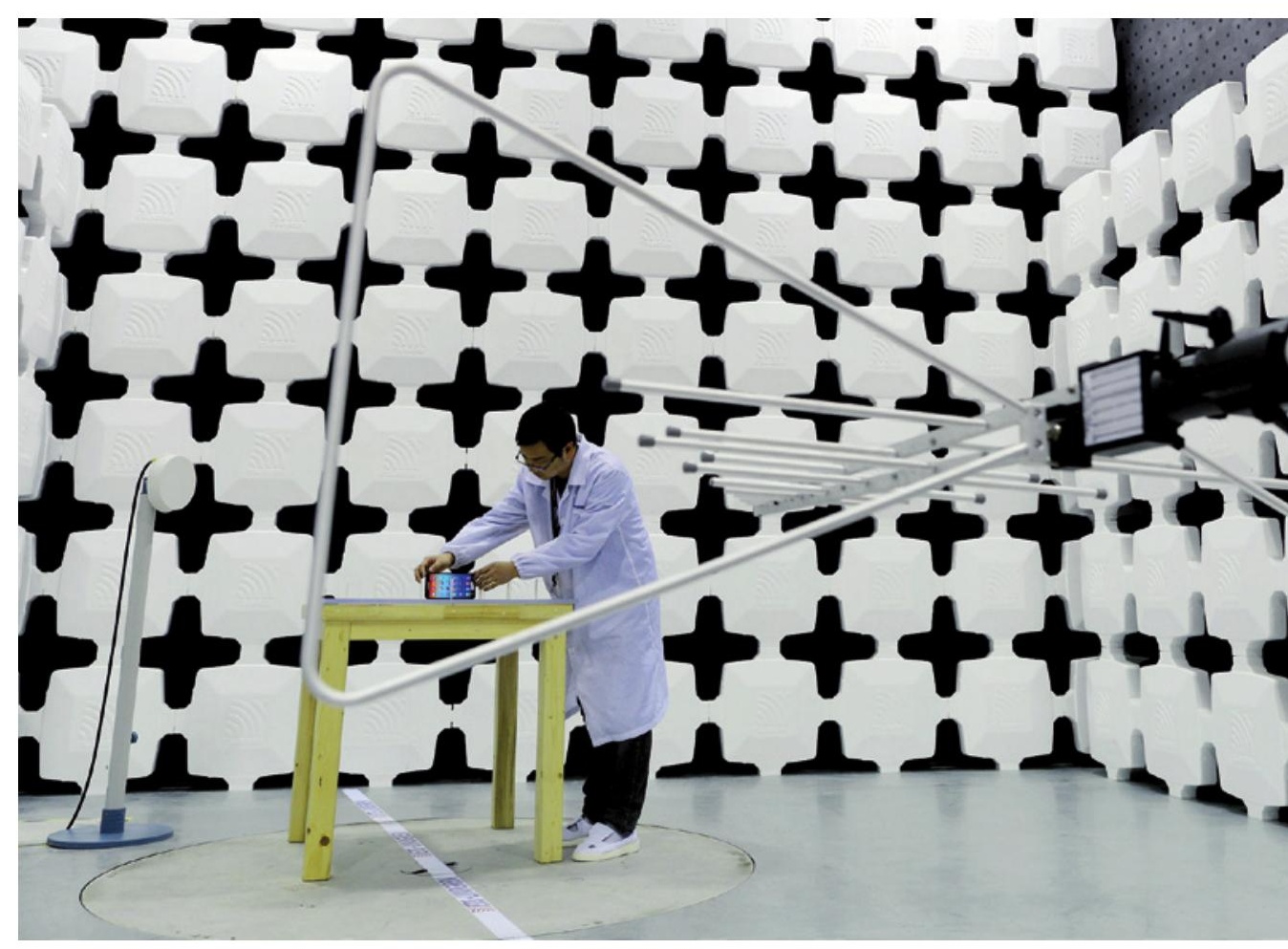

Research at computer firm Lenovo is helping to drive China's rising R\&D spending.

\section{RESEARCH FUNDING}

\section{China tops Europe in R\&D intensity}

Reforms to commercial and academic research systems still needed despite reaching spending milestone, say scientists.

\section{BY RICHARD VAN NOORDEN}

$\mathrm{B}$ y pouring cash into science and technology faster than its economy has expanded, China has for the first time overtaken Europe on a key measure of innovation: the share of its economy devoted to research and development (R\&D).

In 2012, China invested $1.98 \%$ of its gross domestic product (GDP) into R\&D - just edging out the 28 member states of the European Union (EU), which together managed 1.96\%, according to the latest estimates of research intensity, to be released this month by the Parisbased Organisation for Economic Co-operation and Development (OECD).

The figures show that China's research intensity has tripled since 1998, whereas Europe's has barely increased (see 'Shooting star'). The numbers are dominated by business spending, reflecting China's push in the manufacturing and information- and communication-technology industries.

James Wilsdon, a science-policy analyst at the University of Sussex in Brighton, UK, says that China's R\&D juggernaut is “astonishing", considering that the entire system emerged only after the end of the Cultural Revolution in 1976. In absolute terms, China's R\&D spending is still almost one-third lower than that of Europe, but the new figures are "a significant milestone", says Wilsdon.

The reorientation of China's economy displays its soaring ambition. However, money does not buy innovation. Despite success in some areas, notably high-speed rail, solar energy, supercomputing and space exploration, leaders in China are concerned that innovation is lacking, say science-policy analysts. "Chinese leaders would like something equivalent to a Nobel prize, or a worldclass product similar to an iPhone," says Denis Simon, an expert on Chinese science and innovation at Arizona State University 